\title{
Herniation of an Antral Polyp through an Oro-Antral Fistula
}

\author{
Ming Zhao', , Ikuko Ogawa ${ }^{3}$, Masaki Kiyomihara ${ }^{4}$ and Takashi Takata ${ }^{2,3}$ \\ ${ }^{1}$ Department of Periodontics / Prevention / Geriatrics, University of Michigan School of Dentistry, Ann Arbor, USA \\ ${ }^{2}$ Department of Oral Maxillofacial Pathobiology, Division of Frontier Medical Science, Graduate School of Biomedical Sciences, \\ Hiroshima University, Hiroshima, Japan \\ ${ }^{3}$ Clinical Laboratory, Hiroshima University Dental Hospital, Hiroshima, Japan \\ ${ }^{4}$ Department of Oral and Maxillofacial Surgery, Division of Cervico-Gnathostomatology, Graduate School of Biomedical Sciences, \\ Hiroshima University, Hiroshima, Japan
}

\begin{abstract}
Zhao M, Ogawa I, Kiyomihara M and Takata T. Herniation of an antral polyp through an oroantral fistula. Oral Med Pathol 2003; 8: 101-103, ISSN 1342-0984

Epulis, a discrete sessile or pedunculated mass of fibrous tissue on the gingiva caused by chronic inflammation, is a common lesion. However, other lesions may occasionally exhibit exophytic growth and they must be differentiated from epulis.

A 38-year-old Japanese female noticed a pedunculated soft mass on the alveolar ridge of the right maxillary third molar shortly after extraction of the tooth. The mass, which had periods of exacerbation and quiescence, was excised under a tentative diagnosis of epulis. Because histopathology revealed edematous, inflamed antral mucosa with a covering of ciliated columnar epithelium, a final diagnosis of herniation of antral polyp through an oro-antral fistula was established. Although a rare occurrence, herniation of antral polyp should be included in the differential diagnosis of exophytic lesions on the maxillary molar alveolar ridge if there is a history of dental extraction at the concerned area.
\end{abstract}

Key words: herniation, antral mucosa, tooth extraction, epulis, periodontics

Correspondence: Takashi Takata, Department of Oral Maxillofacial Pathobiology, Division of Frontier Medical Science, Graduate School of Biomedical Sciences, Hiroshima University, 1-2-3 Kasumi, Minami-ku, Hiroshima 734-8553, Japan

Phone: +81-82-257-5631, Fax :+81-82-257-5619, E-mail: ttakata@hiroshima-u.ac.jp

\section{Introduction}

An oro-antral fistula, an unnatural communication between the mouth and the maxillary sinus after tooth extraction, is not uncommon, but herniation of an antral polyp through an oro-antral fistula is very rare. Because of its rarity and macroscopic resemblance to an inflammatory overgrowth of gingiva, this lesion may be misdiagnosed as an epulis. Here we report a case of herniation of an antral polyp in order to call the attention of dentists to this lesion.

\section{Case Report}

A 38-year-old Japanese female was referred to our dental hospital for the evaluation of a pedunculated soft tissue mass on the alveolar ridge in the area of the missing upper right third molar. About one and a half years before, she had visited a dentist because of pain and swelling in the right maxilla. In addition, she had a nose obstruction. Infection of the right maxillary sinus was doubted and the right upper third molar was extracted. Communication between antrum and oral cavity was noticed right after the extraction, but no special treat- ment was made. The mass enlarged gradually and diminished spontaneously several times. She visited our hospital for the reappearance of the mass.

Intraoral examination revealed a pedunculated, exophytic soft tissue mass with a dark red surface on the alveolar ridge in the area of the missing third molar, one part of which protruded to the buccal aspect of the second maxillary molar (Fig.1). The mass was about

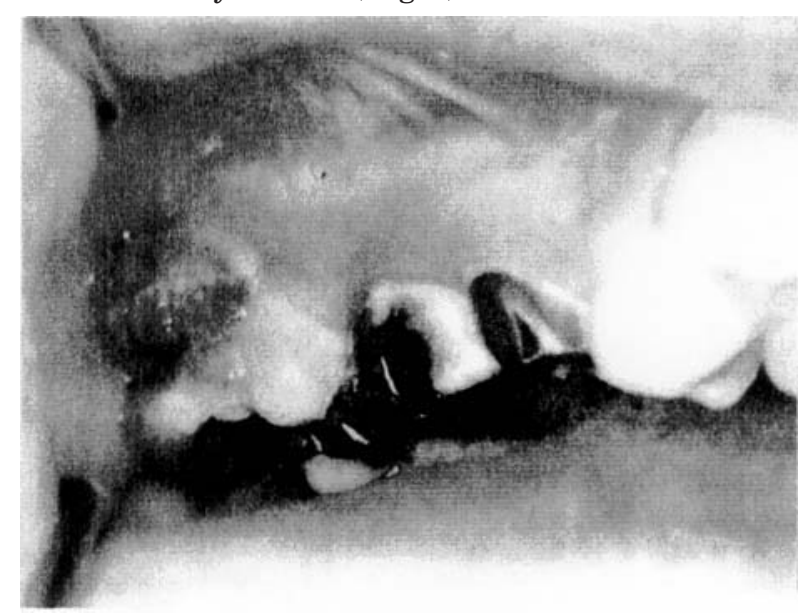

Fig 1: An exophytic soft tissue mass with a granular surface protrudes from the alveolar ridge in the area of the missing third molar to the buccal aspect of the second maxillary molar. 
$101020 \mathrm{~mm}$ in size, elastic and nontender to palpation. Neither nasal obstruction nor rhinorrhea was observed. Radiological examination revealed opacity of the right maxillary sinus, suggesting chronic sinusitis (Fig. 2).

Since a definite diagnosis could not be established clinically, excision of the mass was performed under a tentative clinical diagnosis of epulis granulomatosum. Upon excision of the mass, the communication between the extraction socket and the antrum was seen, and a small amount of purulence was discharged from the sinus. After antibiotic therapy and irrigation were administered postoperatively, the oro-antral fistula in the extraction socket shrank nearly to closure and no reappearance of the mass has been noticed in a 16-month followup.

Microscopic examination of the excised mass revealed an edematous, inflamed antral mucosa surface identified by a covering of ciliated columnar epithelium (Figs. 3a, b). There were focal areas of squamous metaplasia and broad zones of ulceration with heavy surface bacterial colonization (Fig. 3c). The connective tissues contained a fibrinous exudate heavily laden with neutrophils, lymphocytes and plasma cells. A final diagnosis of herniation of an antral polyp through an oro-antral fistula was established.

\section{Discussion}

An oro-antral fistula is an unnatural communication between the oral cavity and the maxillary sinus resulting most commonly from the extraction of teeth. Although oro-antral fistula after tooth extraction is not an uncommon occurrence, herniation of the antral mucosa through an oro-antral fistula is very rare. Only five reported cases were retrieved from the English literature (1-4).

Because of the close relationship between the root apices of maxillary molars and the antrum, post-extraction oro-antral opening is not uncommon, but it may go unrecognized immediately by the dentist (1). In most cases, the resultant communication undergoes a spontaneous closure following the organization of the blood clot filling the socket. But an oro-antral fistula may ensue when the opening is too large, the alveolar bone is too thin, infection is present in the sinus or the periapical tissue, or the patient's activity results in an oro-antral pressure difference.

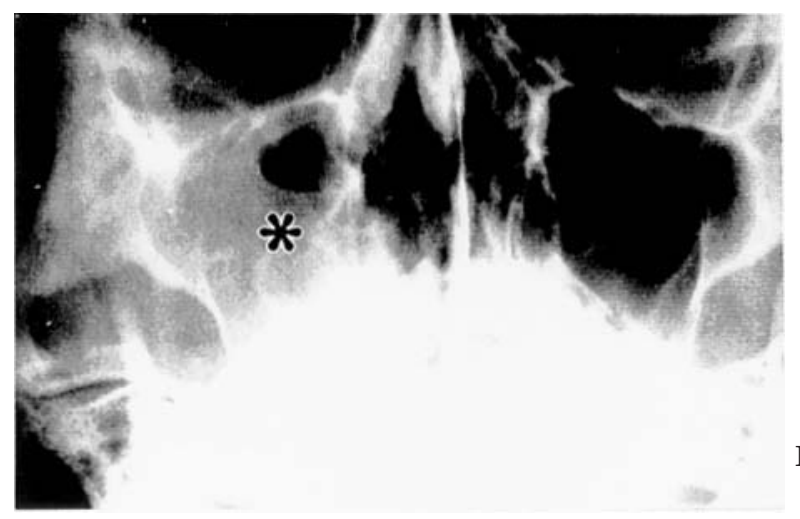

Fig 2: Radiological features. Waters view showing right maxillary sinus opacity $(*)$.

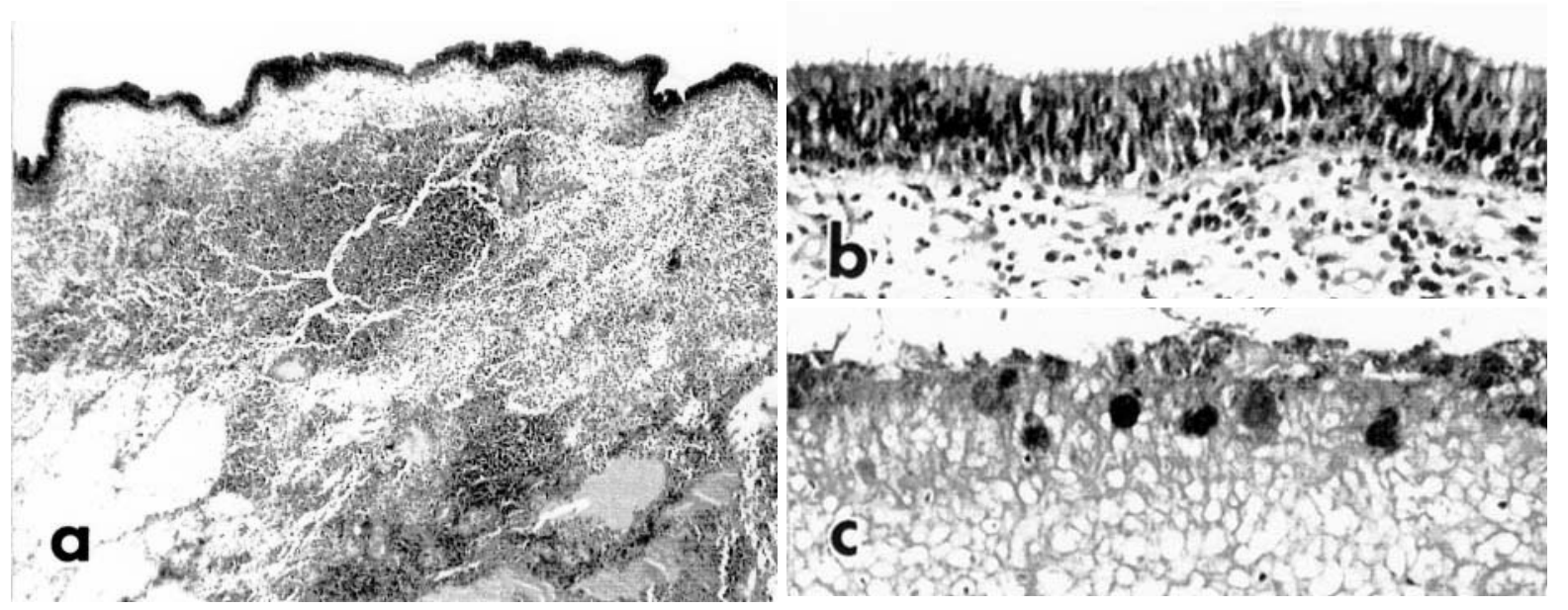

Fig 3: Histopathology of the lesion. a, The exophytic mass is composed of an edematous connective tissue with a dense inflammatory cell infiltration. b, Ciliated columnar respiratory type epithelium covering the surface. c, Surface ulceration with heavy bacterial colonization. HE, (Magnification, a: 40, b: 200, c: 200) 
In the present case, the clinical presentation of this mass suggested an inflammatory hyperplastic lesion or a gingival tumor, most probably an epulis granulomatosum. Since loss of bone from the antral floor was observed radiologically, an antral polyp protruding into the oral cavity through the extraction socket should be included in the differential diagnosis. However, this possibility was overlooked during clinical examination mainly because of not knowing of this lesion.

Maxillary sinusitis is often due to the direct extension of dental infections, and it is also a common complication of oro-antral fistula which is not treated in time (3). In addition, maxillary sinusitis may also originate from infectious diseases such as the common cold, influenza and exanthematous diseases. In long-term chronic maxillary sinusitis, the mucosal lining of the antrum often shows remarkable thickening and the development of antral polyps (3). In the present case, the antral inflammation was considered to precede the extraction of the third maxillary molar from the clinical symptoms and X-ray examination. The communication between the socket and antrum occurred during the tooth extraction, but the sinusitis and oro-antral fistula were not properly treated in time, so the inflammatory antral mucosa was swollen so severely that the maxillary ostium was stopped up and the evacuation of exudate was blocked. With the exacerbation of antral inflammation and increase of the intra-antral pressure, the antral polyp protruded through the oro-antral fistula into oral cavity to form the herniation.
Although it is uncommon, herniation of antral polyps should be included in a differential diagnosis of exophytic lesions on the maxillary molar alveolar ridge if there is a history of tooth extraction at the concerned area. The nature of such a lesion may be suggested by the history, clinical appearance and radiographic findings. The treatment of choice is surgical removal of the lesion and closure of the oro-antral fistula under the complete control of sinusitis.

\section{References}

1. Norman JE and Craig G. Oroantral fistula: an analysis of 100 cases. Oral Surg Oral Med Oral Pathol 1971; 31: 734-44.

2. Vizuete JR, Ross VA and Craig RM. An oral soft tissue lesion associated with a sinus mass. J Am Dent Assoc 1985; 110: 535-6.

3. Amaratunga NA. Oro-antral fistulae. A study of clinical, radiological and treatment aspects. Br J Oral Maxillofac Surg 1986; 24: 433-7.

4. Raut VV and Bhattacharya AK. Expansion of the maxillary antrum by an antrol-choanal polyps (a case report). $J$ Postgrad Med 1991; 37: 123-4.

5. Shultz RE, Theisen FC and Dunlap CL. Herniation of the antral membrane through an extraction site. Report of a case. Oral Surg Oral Med Oral Pathol 1991; 71: 280-2.

6. Takeda Y. Herniation of an antral polyp through an oroantral fistula. Ann Dent 1992; 51: 26-8.

(Accepted for publication April 4, 2003) 